\begin{tabular}{llll}
\multicolumn{4}{l}{ Abstract PWE-060 Table 1} \\
\hline \% & 2010 & 2012 & \\
\hline CBF & 15.2 & 23.0 & OR $=1.67$, Cl: $1.61-1.72$ \\
CS & 21.3 & 23.3 & OR $=1.12$, Cl: $1.09-1.16$ \\
HBF & 14.1 & 10.1 & OR $=0.68$, Cl: $0.66-0.71$ \\
HS & 41.0 & 31.1 & OR $=0.65$, Cl: $0.63-0.67$ \\
EMR & 8.5 & 12.5 & OR $=1.55$, Cl: $1.48-1.62$ \\
\hline
\end{tabular}

practice. We aimed to examine the techniques employed for removal of $<10 \mathrm{~mm}$ polyps in relation to polyp characteristics, completeness of excision, safety and changes over time.

Methods Data relating to removal of polyps $<10 \mathrm{~mm}$ between Jan 2010 and Dec 2012 were retrieved from the national Bowel Cancer Screening Programme (BCSP) database. Categorical data was compared using $x^{2}$.

Results 147174 polyps were removed during 62679 colonoscopies. A range of techniques was used (cold biopsy forceps (CBF) 19.7\%, cold snare (CS) 22.1\%, hot biopsy forceps (HBF) $12.2 \%$, hot snare (HS) 35.1\%, EMR 10.9\%).

EMR was used more frequently in the right colon compared to the left (14.3 vs. $8.3 \%$, OR $=1.84,95 \% \mathrm{CI}: 1.78-1.90)$.

Most pedunculated polyps were removed using HS; this was lower in the right vs. left colon $(69.6$ vs. $88.3 \%$, OR $=0.30$, CI: $0.28-0.33)$. CS was most common for non-pedunculated polyps in the right colon $(29.8$ vs. $19.0 \%$ in left, $\mathrm{OR}=1.81 \mathrm{CI}$ : 1.76-1.85); whereas most common in the left colon was HS (34.8 vs. $22.5 \%$ in right, $\mathrm{OR}=1.84 \mathrm{CI}: 1.79-1.88$ ).

Surgeons were more likely than physicians to use diathermy irrespective of site or morphology (65.6 vs. $56.5 \%, \mathrm{OR}=1.46 \mathrm{CI}$ : 1.43-1.5).

In $60 \%$ of polyps removed completeness of excision was not histologically assessable. 21.2\% were completely excised, 5.8\% incomplete and $13 \%$ not stated. For non-pedunculated polyps, histologically-confirmed complete excision was more common after EMR (23.4 vs. $6.2 \%$, OR $=1.16, \mathrm{CI}: 1.08-1.25) \mathrm{com}-$ pared to other techniques (CBF 17.7\%, CS 15.1\%, HBF 19.1\%, HS 21.5\%); for pedunculated polyps it was more common after EMR (42.3\%) and HS (42.0\%).

Complications were rare for colonoscopies (45227) where only polyps $<10 \mathrm{~mm}$ were removed. $12(0.03 \%)$ bleeding episodes required transfusion; rates for single and multiple polypectomy cases were 0.01 and $0.04 \%$ respectively (OR $=5.01, \mathrm{CI}: 1.10-22.8)$. The HS technique was most commonly used. There were $16(0.04 \%)$ perforations; $0.02 \%$ for single vs. $0.05 \%$ for multiple polypectomies $(\mathrm{OR}=2.20, \mathrm{CI}: 0.77-6.34, \mathrm{p}=0.13)$. No technique dominated for single compared with HS for multiple polypectomies.

Between 2010 and 2012, use of CBF, CS and EMR increased, whereas HBF and HS decreased $(\mathrm{p}<0.01)$

Conclusion The removal of polyps $<10 \mathrm{~mm}$ within the BCSP is safe, but histological evidence of completeness of excision is poor with all techniques. Wide variations in practice reflect the lack of evidence guiding these decisions, although use of cold resection techniques has increased over time

Disclosure of Interest None Declared.

\section{PWE-061 SPLIT-DOSE MOVIPREP (LOW VOLUME PEG) AND AFTERNOON COLONOSCOPY: A STEP IN THE RIGHT DIRECTION}

S Ghuman*, P George, K Jones, H Khan. Gastroenterology, Wrexham Maelor Hospital, Wrexham, UK

10.1136/gutinl-2014-307263.321

\begin{tabular}{|c|c|c|c|c|c|}
\hline Symptomatic cases & Picolax & $\begin{array}{l}\text { Split dose } \\
\text { Moviprep }\end{array}$ & $\begin{array}{l}\text { Sinele dose } \\
\text { Moviprep }\end{array}$ & $\begin{array}{l}\text { Momine } \\
\text { list }\end{array}$ & $\begin{array}{l}\text { Afternoon } \\
\text { list }\end{array}$ \\
\hline Total cases & 50 & so & 50 & 78 & 72 \\
\hline Caecal intubation & $46(92 \%)$ & $48(96 \%)$ & $44(88 \%)$ & $72(92.3 \%)$ & $66(91.6 \%)$ \\
\hline Number of polyps detected & 17 & 14 & 47 & 37 & 41 \\
\hline Good bowel prep & $24(48 \%)$ & $28(56 \%)$ & $19(38 \%)$ & $24(30.8 \%)$ & $47(65.3 \%)$ \\
\hline Satisfactory bowel prep & $19(38 \%)$ & $19(38 \%)$ & $21(42 \%)$ & $40(51.3 \%)$ & $19(26.4 \%)$ \\
\hline Poor bowel prep & $7(14 \%)$ & $3(6 \%)$ & $10(20 \%)$ & $14(17.9 \%)$ & $6(8.3 \%)$ \\
\hline \multicolumn{6}{|l|}{ Screening cases } \\
\hline Total cases & 50 & 50 & 50 & \multirow{6}{*}{\multicolumn{2}{|c|}{$\begin{array}{l}\text { All screening cases were } \\
\text { done in the morning list }\end{array}$}} \\
\hline Caecal intubation & $47(94 \%)$ & $48(96 \%)$ & $47(94 \%)$ & & \\
\hline Number of polyps detected & 81 & 64 & 73 & & \\
\hline Good bowel prep & $11(22 \%)$ & $37(74 \%)$ & $25(50 \%)$ & & \\
\hline Satisfactory bowel prep & $34(68 \%)$ & $12(24 \%)$ & $22(44 \%)$ & & \\
\hline Poor bowel prep & $5(10 \%)$ & $1(2 \%)$ & $3(6 \%)$ & & \\
\hline
\end{tabular}

\section{Abstract PWE-061 Figure 1}

Introduction Good bowel preparation is essential for optimal mucosal visualisation during colonoscopy. The aim of this retrospective study was to evaluate the efficacy of three types of bowel preparation - Picolax (sodium picosulphate), single dose Moviprep and split-dose Moviprep.

Methods Two groups of patients; bowel cancer screening and symptomatic patients - who underwent colonoscopy at our institution over a 12-month period were identified. Within the two groups, 50 patients receiving each type of bowel preparation were selected providing a total of 300 . Data collected included subjective endoscopist rating of bowel preparation quality (good, satisfactory, poor), depth of insertion, timing of endoscopy and polyp detection.

Results In symptomatic patients, 94\% prescribed split-dose Moviprep had good or satisfactory bowel preparation with an unadjusted caecal intubation rate of $96 \%$. $80 \%$ prescribed single dose Moviprep and $84 \%$ prescribed Picolax received the same rating with a caecal intubation rate of 88 and $92 \%$ respectively. More afternoon colonoscopies received a 'good' bowel preparation rating $(65.3$ vs $30.8 \%$, p value $<0.001)$ and more polyps (52.6 vs $47.4 \%$ ) were detected regardless of preparation type. Moviprep was associated with the highest polyp detection rate (61 vs $34 \%$, p value 0.03 ). In screening patients, $98 \%$ prescribed split-dose Moviprep had good or satisfactory bowel preparation. 94\% prescribed single dose Moviprep and 90\% prescribed picolax achieved the same rating. There was no significant difference in caecal intubation or polyp detection within the screening group.

Conclusion Split-dose Moviprep and colonoscopy performed in the afternoon are two independent factors facilitating better bowel cleansing and higher polyp detection.

Disclosure of Interest None Declared.

\section{PWE-062 TERMINAL ILEAL INTUBATION OVER A FIVE YEAR PERIOD; WAS IT USEFUL?}

S Ghuman*, H Khan. Gastroenterology, Wrexham Maelor Hospital, Wrexham, UK

\subsection{6/gutjnl-2014-307263.322}

Introduction The value of routine ileoscopy during colonoscopy is unclear, but intubation of the terminal ileum (TI) is considered to be the main method of confirming completeness of colonoscopy. TI intubation rates are variable and intubation is often omitted due to time constraints and the perception of little added diagnostic value. Our aim was to assess the diagnostic yield of TI intubation during colonoscopies at our institution.

Methods A retrospective study was undertaken at our institution. Colonoscopy data over a 5 year period $\left(1^{\text {st }}\right.$ October 2007 to $30^{\text {th }}$ September 2012), were retrieved from the Endoscopy Reporting System database (Unisoft, Enfield, UK). Patients with 
ileo-caecal resection were excluded. Demographic data, TI pathology (endoscopic and histopathologic) and indications for colonoscopy were analysed.

Results 8016 colonoscopies were performed with a caecal intubation rate of $90.3 \%$. The endoscopists were of different grades including gastroenterologists, colorectal surgeons and a nurse endoscopist. 206 with ileo-caecal resection were excluded. Mean age was 61 with a female preponderance at $52.6 \%$. The TI $\mathrm{w}$ as intubated in 1845 (23.5\%). Endoscopic TI pathology was identified in 42 patients (2.3\%). Histology was available for 31, of which $23(1.3 \%)$ had confirmed histological abnormalities. Diagnoses on ileoscopy included one adenocarcinoma, one carcinoid tumour, one metastatic malignant melanoma and 20 with terminal ileitis, of which, 6 had histological Crohn's disease. The most common indications in those with TI pathology were diarrhoea (15), abdominal pain (8) and rectal bleeding (8).

Conclusion Although the overall diagnostic yield was low, TI intubation identified significant pathologies requring further action, including three malignancies. Ileoscopy at colonoscopy is a simple manoeuvre, which, apart from quality assurance can identify important pathology. The most common indication in those with confirmed TI pathology was diarrhoea, therefore ileoscopy may have added diagnostic value in this context.

Disclosure of Interest None Declared.

\section{PWE-063 COLONOSCOPY IN THE OVER NINETIES: HOW USEFUL AND SAFE IS IT?}

SK Butt*, A Chitembwe, K Besherdas. Gastroenterology, Chase Farm Hospital, London, UK

10.1136/gutjnl-2014-307263.323

Introduction Colonoscopy is accepted as the gold standard imaging modality of the colon for investigation of colonic symptoms including altered bowel habit, anaemia, rectal bleeding and for the detection of colorectal cancer. The incidence of pathological findings within the colon in particular colorectal cancer increases with age, and the detection of these cancers comprise a major role of colonoscopy in the elderly population. However, colonoscopy in the elderly may be more difficult, as elderly patients are more likely than younger patients to have poor bowel preparations. Furthermore, complications of colonoscopy, in particular cardiovascular, maybe higher in this age group. To date no data exist in colonoscopies performed in those over 90 years of age.

Methods The aim of the study was to assess the diagnostic yield and safety of colonoscopy in the very elderly patients over 90 years of age. A single centre, retrospective analysis of patients over 90 years of age in a district general hospital from north London was performed. The patients were identified using the Unisoft Endoscopy reporting software over a period between June 2006 to March 2013. Data obtained during the study period was scrutinised for indication, sedation administered, quality of bowel prep, findings of procedure, and complications. Results During the study period, 66 colonoscopies were performed out of a total 666 endoscopic procedures in patients over 90 . The indications for colonoscopy included : abnormal CT scan 5/66, altered bowel habit in $17 / 66$, anaemia 24/66, rectal bleeding $15 / 66$, colonic obstruction $3 / 66$, previous polyp/ colon cancer 4/66, abdominal mass 1/66. 18/66 patients had poor bowel prep. Findings included normal in $18 / 66$, colo-rectal cancer in $13 / 66$ patients, colonic polyps in $5 / 66$, ischaemic colitis
$1 / 66$, angiodysplasia 2/66, diverticulosis in $31 / 66$. Sedation administered varied from none in $4 / 66$, to midazolam $5 \mathrm{mg}+$ pethidine $50 \mathrm{mg}$ in $2 / 66$. There were no complications from the colonoscopy in 66 patients within the study.

Conclusion The data from this study demonstrates that colonoscopies can be performed safely in patients over 90 years of age with no increase in procedure related mortality. Despite poor bowel preparation encountered in $28 \%$, the diagnostic yield of pathology in patients over 90 having colonoscopy was very high with an abnormality in 48/66 (72\% in this study). Colonoscopy in the very elderly is very safe and worthwhile and clinicians should not be reluctant in referring those over 90 years of age for colonoscopic examination.

Disclosure of Interest None Declared.

\section{PWE-064 MANAGEMENT OF LARGE COLONIC POLYPS IN THE SEVERN DEANERY HOSPITALS: AN AUDIT OF CURRENT PRACTICE}

${ }^{1}$ SW Dixon*, ${ }^{1} \mathrm{~T}$ Valliani, ${ }^{2} \mathrm{~F}$ Fayyaz, ${ }^{3} \mathrm{D}$ Tate, ${ }^{4} \mathrm{E}$ Arthurs, ${ }^{4} \mathrm{~L}$ Lim, ${ }^{4} \mathrm{~A}$ Dhanda, ${ }^{1} \mathrm{M}$ Lockett. ${ }^{1}$ Gastroenterology, North Bristol NHS Trust, Bristol, UK; ${ }^{2}$ Gastroenterology, Royal United Hospitals, Bath, Bath, UK; ${ }^{3}$ Gastroenterology, Gloucestershire Hospitals NHS Trust, Gloucester, UK; ${ }^{4}$ Gastroenterology, University Hospitals Bristol NHS Trust, Bristol, UK

\subsection{6/gutjnl-2014-307263.324}

Introduction Large $(>2 \mathrm{~cm})$ colonic polyps present a challenge to the colonoscopist. The British Colorectal Cancer Screening Programme (BCSP) recently drafted guidelines to standardise their management. We compared our current practice with the proposed guidelines.

Methods This was a retrospective audit in four South-West hospitals with comparison between screening and non-screening patients. Patients were identified using clinical coding. Case notes were reviewed. Polyps were scored using SMSA system to standardise difficulty of endoscopic resection. Data was compared against 9 auditable outcomes.

Results 104 cases were identified (24 BCSP, 80 symptomatic). There was no significant difference in mean size $(2.9 \mathrm{~cm}$ BCSP, $2.7 \mathrm{~cm}$ symptomatic, $\mathrm{p}=0.14)$ or mean SMSA grading $(2.8$ BCSP, 2.9 symptomatic, $\mathrm{p}=0.46) .6$ polyps were malignant $(1$ BCSP; 5 symptomatic); all had position marked by tattoo. 1 malignant polyp was resected endoscopically in the symptomatic group. Mean time to definitive resection was 34 and 30 days (BCSP and symptomatic respectively). Recurrence of adenoma at EMR site was low at 3 months (0/22 BCSP, 1/37 symptomatic) and 12 months (0/22 BCSP, 2/37 symptomatic). However fewer EMR sites were checked if the index endoscopy was performed by a non-BCS colonoscopist (10/13 vs. 8/24). Complication rates were low: 1 haemorrhage requiring admission (symptomatic group); 0 perforations. 17 benign polyps were referred for surgery ( 3 BCSP, 14 symptomatic; $p=0.49$ ). In both groups the mode SMSA score was 4 . Benign polyps were referred directly for surgery; only those polyps with malignant histology were discussed at a formal mutli-disciplinary meeting. There were no deaths or signficant morbidity associated with surgery for bening polyps.

Conclusion Overall management was comparable to draft guidelines. The frequency of large polyps outside BCSP indicates that non-BCS endoscopists will gain experience with these lesions. Approximately $17 \%$ of benign polyps were referred for surgery, similar to other series. However, none had been discussed with local EMR experts. Consequently a large polyp referral pathway has been established at two centres within the region. 